\title{
Fatores relacionados à iniciação das práticas sexuais precoces na adolescência: uma revisão integrativa
}

\author{
Factors related to the initiation of early sexual practices in adolescence: an integrative review \\ Factores relacionados con la iniciación de prácticas sexuales tempranas en la adolescência: una \\ revisión integradora
}

Recebido: 29/10/2021 | Revisado: 06/11/2021 | Aceito: 07/11/2021 | Publicado: 12/11/2021

\author{
Wallacy Jhon Silva Araújo \\ ORCID: https://orcid.org/0000-0001-7916-1250 \\ Universidade Federal de Pernambuco, Brasil \\ E-mail: wallacyjhon@outlook.com \\ Mariana Isabel Alexandre Moura \\ ORCID: https://orcid.org/0000-0001-8750-0224 \\ Universidade Federal de Pernambuco, Brasil \\ E-mail: marianaisabel8@gmail.com \\ Gabriela Rodrigues Bragagnollo \\ ORCID: https://orcid.org/0000-0003-1480-8046 \\ Universidade de São Paulo, Brasil \\ E-mail: gabriela.rodrigues.bragagnollo@usp.br \\ Rosangela Andrade Aukar De Camargo \\ ORCID: https://orcid.org/0000-0002-4872-2331 \\ Universidade de São Paulo, Brasil \\ E-mail: rcamargo@eerp.usp.br \\ Estela Maria Leite Meirelles Monteiro \\ ORCID: https://orcid.org/0000-0002-5736-0133 \\ Universidade Federal de Pernambuco, Brasil \\ E-mail: estelameirellesufpe@gmail.com
}

\begin{abstract}
Resumo
Objetivo: identificar evidências científicas acerca dos principais fatores relacionados ao início das práticas sexuais precoces na adolescência. Método: revisão integrativa de artigos publicados em português, inglês ou espanhol, nas bases de dados LILACS, BDENF, MedLine, CINAHL e Web of Science. A questão norteadora foi: quais os principais fatores relacionados com a iniciação das práticas sexuais precoces na adolescência. Foram utilizados os descritores Adolescent, Sexuality, Sexual Behavior, Sexual Intercourse e Coitus, sendo selecionados 38 artigos para análise. Resultados: os resultados deram origem à três classes: Vinculações familiares e entre os pares; Situações de vulnerabilidade e Ações promotoras de saúde. As classes evidenciaram que os fatores relacionados à iniciação das práticas sexuais precoces na adolescência apresentam relações com cenários diversos, capazes de influenciar de forma positiva ou negativa nas decisões do adolescente acerca do início da sua vida sexual. Conclusão: o estudo permitiu uma reflexão crítica acerca dos fatores que contribuem para determinar a iniciação das práticas sexuais precoces na adolescência, como também apreender estratégias protetivas, que concorrem para adiar o início das práticas sexuais.

Palavras-chave: Adolescente; Sexualidade; Comportamento sexual; Relação sexual; Promoção da saúde.
\end{abstract}

\begin{abstract}
Objective: to identify scientific evidence about factors related to the beginning of early sexual practices in adolescence. Method: Integrative review of articles published in Portuguese, English or Spanish, in the LILACS, BDENF, MedLine, CINAHL and Web of Science databases. The descriptors Adolescent, Sexuality, Sexual Behavior, Sexual Intercourse and Coitus were used, with thirty-eight articles selected for analysis. Results: in the selected studies, thirty-seven were quantitative and one qualitative. The results gave rise to three classes: Family and peer ties; Vulnerable situations and health-promoting actions. The classes showed that the factors related to the initiation of early sexual practices in adolescence are related to different scenarios, capable of influencing in a positive or negative way the adolescents' decisions about the beginning of their sexual life. Conclusion: The study allowed a critical reflection on the factors that contribute to determine the initiation of early sexual practices in adolescence, as well as to learn protective strategies, which contribute to postpone the beginning of sexual practices.
\end{abstract}

Keywords: Adolescent; Sexuality; Sexual behavior; Sexual intercourse; Health promotion. 


\begin{abstract}
Resumen
Objetivo: identificar evidencia científica sobre los principales factores relacionados con el inicio de prácticas sexuales precoces en la adolescencia. Método: revisión integradora de artículos publicados en portugués, inglés o español, en bases de datos LILACS, BDENF, MedLine, CINAHL y Web of Science. La pregunta orientadora fue: ¿Cuáles son los principales factores relacionados con el inicio de prácticas sexuales tempranas en la adolescencia? Se utilizaron los descriptores Adolescente, Sexualidad, Conducta sexual, Relaciones sexuales y Coito, y se seleccionaron 38 artículos para su análisis. Resultados: los resultados dieron lugar a tres clases: lazos familiares y de pares; Situaciones vulnerables y acciones de promoción de la salud. Las clases mostraron que los factores relacionados con el inicio de prácticas sexuales tempranas en la adolescencia tienen relaciones con diferentes escenarios, capaces de influir positiva o negativamente en las decisiones de los adolescentes sobre el inicio de su vida sexual. Conclusión: el estudio permitió una reflexión crítica sobre los factores que contribuyen a determinar el inicio de prácticas sexuales tempranas en la adolescencia, así como aprehender estrategias protectoras, que contribuyen a retrasar el inicio de prácticas sexuales.
\end{abstract}

Palabras clave: Adolescente; Sexualidad; Conducta sexual; Relaciones sexuales; Promoción de la salud.

\title{
1. Introdução
}

O período da adolescência é caracterizado pela transição da infância para a fase de autonomia civil adulta, sendo marcada pela busca da maturação física, social e psicológica com estruturação da personalidade do indivíduo para aquisição de características de adulto (Faria; Martins, 2016; Genz; Meincke; Carret; Corrêa; Alves, 2017). Na construção das características psicossociais do ser humano, a sexualidade está norteada pelas questões relacionais afetivas, que envolve gênero, diversidade sexual, vínculo amoroso, desejo e o prazer, porém, estes fatores podem exercer influências negativas no equilíbrio psicossocial do adolescente (Alves et al., 2021).

Na compreensão ampliada de sexualidade, encontram-se as relações de amizade, consideradas como um espaço destinado ao desenvolvimento de habilidades sociais necessárias para o aperfeiçoamento das relações entre os pares, como o das relações amorosas e sexuais (Barth; Wagner; Levandowski, 2017).

A sexarca precoce constitui-se como uma tendência principalmente nos países em desenvolvimento, que não possuem programas de Educação Sexual (ES) consolidados nas escolas, como é o caso do Brasil, ocorrendo em adolescentes com menos de 13 anos de idade (Spinola, 2020). A Iniciação Sexual Precoce (ISP) é considerada uma situação de vulnerabilidade, aumentando as chances de adquirir Infecções Sexualmente Transmissíveis (IST), gravidez não planejada, subjugação, violência e/ou exploração sexual, repercutindo de forma negativa na vida do adolescente (Spinola, 2020).

As dimensões anatômica, fisiológica, social, psicológica e espiritual, que envolvem a ISP e as repercussões desta na vida do adolescente, vem requerer políticas de promoção da saúde propícias a estruturar um processo social, político, ético, histórico e cultural, capaz de fortalecer a autonomia e o poder de decisão para mudanças de comportamentos, através de uma prática transversal e intersetorial na sociedade, ao criar e manter os diálogos entre os diversos indivíduos envolvidos no processo saúde-doença como nas atividades de Educação em Saúde (Campos et al., 2017).

A Educação em Saúde apresenta-se como uma ferramenta, utilizada por profissionais da saúde na orientação sexual, sendo executada principalmente no ambiente escolar, para organizar espaços de reflexão, oportunizando questionamentos sobre postura, tabus, crenças, mitos, valores sociais e culturais, numa perspectiva dialógica, interativa e sistemática (Marçal; Miranda, 2020).

Visto a importância do planejamento de atividades de educação em saúde que concorrem para assegurar o desenvolvimento da personalidade do ser adolescente, considerando a contextualização das singularidades e intersubjetividades voltadas a uma ISP, este estudo tem como objetivo identificar evidências científicas disponíveis na literatura acerca dos fatores relacionados ao início das práticas sexuais precoces na adolescência. 


\section{Metodologia}

Trata-se de uma revisão integrativa, que possibilita a análise de subsídios na literatura de forma ampla e sistemática, difundindo dados científicos produzidos por outros autores, destacando-se a exigência dos mesmos padrões de rigor, clareza e replicação utilizados em estudos primários (Torraco, 2016).

O desenvolvimento desta revisão integrativa seguiu sete etapas distintas: Identificação do tema e seleção da questão de pesquisa; Estabelecimento dos critérios de elegibilidade; Identificação dos estudos nas bases científicas; Avaliação dos estudos selecionados e análise crítica; Categorização dos estudos; Avaliação e interpretação dos resultados e Apresentação dos dados na estrutura da revisão integrativa (Lino et al., 2017).

A questão de pesquisa "quais os principais fatores relacionados com a iniciação das práticas sexuais precoces na adolescência?" foi elaborada por meio da estratégia PICo, sintetizada pelo acrônimo P.I.Co. Sendo que "P” corresponde à população (adolescentes), "I" ao fenômeno de interesse (práticas sexuais precoces) e "Co" ao contexto do estudo (fatores associados (Aromataris; Munn, 2017).

A seleção dos estudos foi composta por artigos obedecendo aos seguintes critérios de inclusão: artigos originais, publicados entre os anos de 2014 e 2018, nos idiomas português, inglês e espanhol, que sejam pesquisas que abranjam adolescentes com idade entre 10 e 19 anos de acordo com a faixa etária proposta pela Organização Mundial de Saúde e que respondam à pergunta norteadora da revisão. Os critérios de exclusão estabelecidos foram produções como teses, dissertações, editoriais, capítulos de livros, revisões da literatura e relatos de experiência.

A coleta dos dados ocorreu no período de julho a novembro de 2018 por dois juízes independentes nas seguintes bases de dados: Literatura Latino-Americana e do Caribe em Ciências da Saúde (LILACS), Base de Dados de Enfermagem (BDENF), Medical Literature Analysis and Retrieval System Online (MEDLINE), Cumulative Index to Nursing e Allied Health Literature (CINAHL), Web od Science Thomson Reuters (WEB OF SCIENCE), por meio da utilização dos Descritores em Ciências da Saúde (DeCS) e no idioma inglês do Medical Subject Headings (MeSH): “Adolescent”, "Sexuality”, "Sexual Behavior", "Sexual Intercourse", "Coitus", sendo realizados cruzamentos duplos e triplos com o booleano AND. Durante a etapa de seleção dos artigos, foi realizada exaustiva leitura dos títulos e resumos para certificarmos que estes atendiam à pergunta norteadora e aos critérios de inclusão e exclusão pré-estabelecidos. Foi encontrado um total de identificadas 5.407 publicações. O rigor metodológico foi verificado por meio do Critical Appraisal Skills Programme (CASP), que classifica os estudos em duas categorias: categoria A - seis a dez pontos, caracterizada como boa qualidade metodológica e viés reduzido e categoria B pontuação mínima de cinco pontos, que representa uma qualidade metodológica satisfatória, mas com potencial de viés aumentado (CASP, 2013). Para esta revisão foram incluídos apenas os estudos classificados em nível A.

Para extração dos dados, foi utilizado um formulário contendo: título, ano de publicação, local do estudo, base de dados, periódico, objetivo, população/amostra, resultado, conclusão e nível de evidência. O nível de evidência dos artigos foi determinado, através da seguinte classificação: Nível 1 - evidências provenientes de revisão sistemática ou metanálise de relevantes ensaios clínicos randomizados controlados ou oriundas de diretrizes clínicas baseadas em revisões sistemáticas de ensaios clínicos randomizados controlados; Nível 2 - evidências derivadas de pelo menos um ensaio clínico randomizado controlado bem delineado; Nível 3- evidências obtidas de ensaios clínicos bem delineados sem randomização; Nível 4 evidências provenientes de estudos de coorte e de caso-controle bem delineados; Nível 5 - evidências originárias de revisão sistemática de estudos descritivos e qualitativos; Nível 6 - evidências derivadas de um único estudo descritivo ou qualitativo; Nível 7 - evidências oriundas de opinião de autoridades e/ou relatório de comitês de especialistas (Melnyk; Fineout-Overholi, 2011). Para análise e o processamento crítico dos dados coletados dos estudos foi utilizado o software IRAMUTEQ (Interface R pour lês Analyses Multidimensionnelles de Textes et de Questionnaires - versão 0.7 alpha 2), para subsidiar a 
análises estatísticas sobre o corpus textual, possibilitando a Classificação Hierárquica Descendente (CHD) e expondo as relações entre as classes para a avaliação e comparação dos estudos (Souza et al., 2018).

\section{Resultados}

A partir da busca de estudos por meio dos cruzamentos duplos e triplos, foram encontrados 5.407 artigos nas bases de dados utilizadas. De acordo com o seguimento das etapas de desenvolvimento da presente revisão, 5.276 artigos foram excluídos de acordo com a leitura dos títulos e 56 artigos excluídos após a leitura dos resumos por não estarem em consonância com a temática ou por não responderem à questão norteadora. Dos 75 artigos restantes, procedeu-se com a leitura dos estudos na íntegra, restando assim, 38 estudos que demonstraram afinidade com o tema proposto no presente estudo (Figura 1).

Figura 1. Fluxograma da seleção dos estudos, segundo o protocolo PRISMA, Recife, PE, Brasil, 2018.

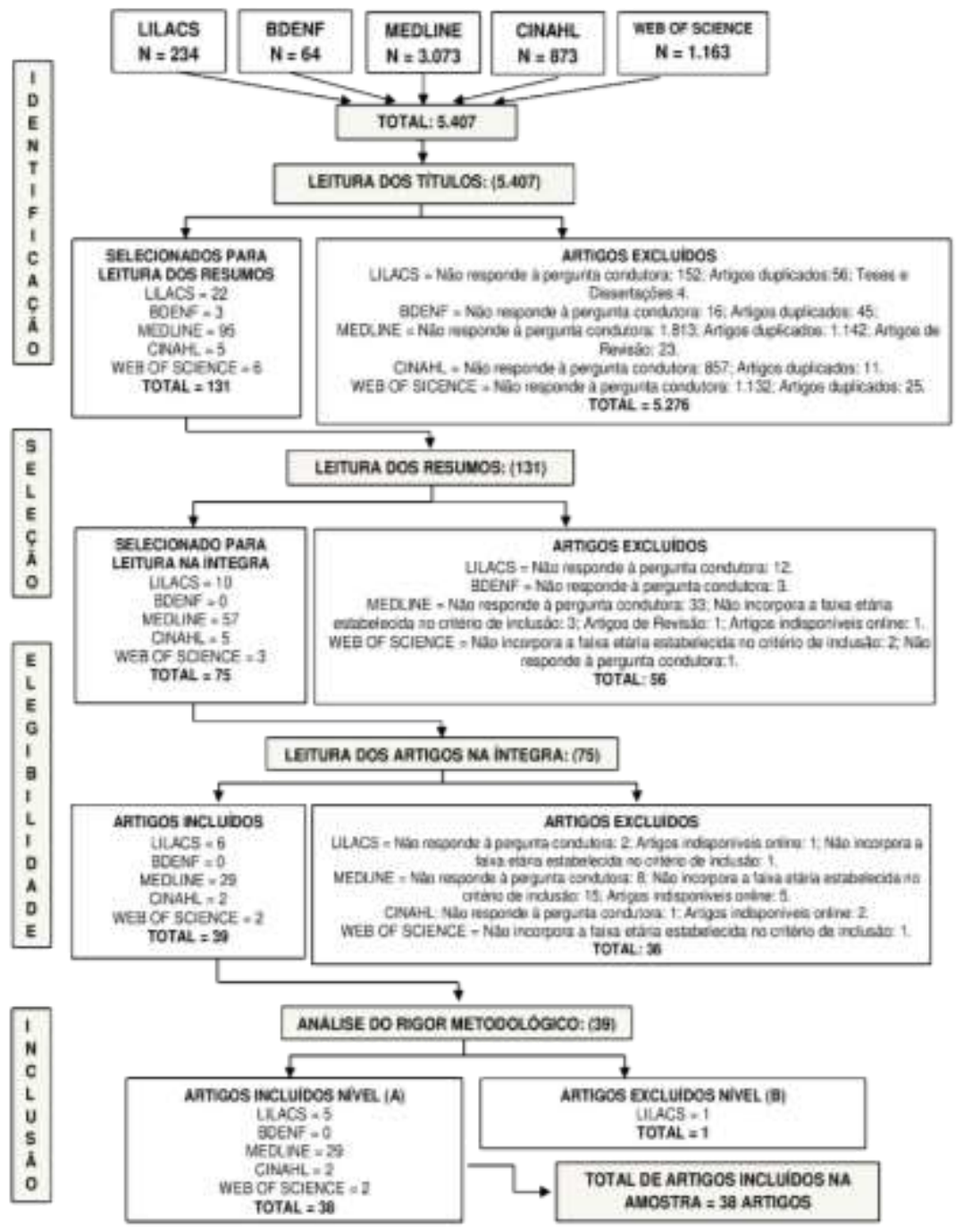

Fonte: Elaborado pelos autores, Recife-PE (2018).

A amostra final foi composta de 38 artigos, sendo vinte nove da MEDLINE, cinco da LILACS, dois da CINAHL e dois da Web of Science. Quanto ao método, apenas um estudo qualitativo e trinta e sete eram quantitativos. Trinta e um com 
nível de evidência 6, seis com nível de evidência 4 e um com nível de evidência $2^{41}$ publicados nos anos de 2014 a 2018 . Uma síntese dos artigos está apresentada no Quadro 1.

Quadro 1 - Síntese dos estudos selecionados. Recife, PE, Brasil, 2018.

\begin{tabular}{|c|c|c|c|}
\hline $\begin{array}{l}\text { Citação / Ano / Base } \\
\text { de dados }\end{array}$ & $\begin{array}{l}\text { Método / } \\
\text { Nível de } \\
\text { evidência }\end{array}$ & Fatores determinantes para ISP & Fatores protetores para ISP \\
\hline $\begin{array}{l}\text { (Useche; Medina; } \\
\text { Ross; Markham, } \\
\text { 2014) } \\
\text { LILACS }\end{array}$ & $\begin{array}{l}\text { Qualitativo } \\
\qquad 6\end{array}$ & $\begin{array}{l}\text { Vivência em um relacionamento amoroso-sentimental; } \\
\text { Curiosidade sexual; Pressão exercida pelo namorado. }\end{array}$ & $\begin{array}{l}\text { Espera por casamento; Medo de } \\
\text { gravidez/paternidade; IST; Influências } \\
\text { familiares; Não ter relacionamento } \\
\text { romântico firme. }\end{array}$ \\
\hline $\begin{array}{l}\text { (Bámaca-Colbert; } \\
\text { Greene; Killoren; } \\
\text { Noah, 2014) } \\
\text { MEDLINE }\end{array}$ & $\begin{array}{l}\text { Longitudinal } \\
\text { Quantitativo } \\
6\end{array}$ & $\begin{array}{l}\text { Estrutura familiar de adolescentes que vivem com pais } \\
\text { adotivos; Experimentação da puberdade precoce. }\end{array}$ & \\
\hline $\begin{array}{l}\text { (Jeremic; Matejic; } \\
\text { Soldatovic; Radenovic, } \\
\text { 2014) } \\
\text { MEDLINE }\end{array}$ & $\begin{array}{l}\text { Quantitativo } \\
6\end{array}$ & $\begin{array}{l}\text { Ter piores médias de notas em ano letivo anterior; Sentir- } \\
\text { se inseguro na escola; Usar bebida alcoólica, tabaco e } \\
\text { maconha. }\end{array}$ & $\begin{array}{l}\text { Programas e intervenções de promoção à } \\
\text { saúde. }\end{array}$ \\
\hline $\begin{array}{l}\text { (Jovic et al., 2014) } \\
\text { MEDLINE }\end{array}$ & $\begin{array}{l}\text { Transversal } \\
\text { Quantitativo } \\
6\end{array}$ & $\begin{array}{l}\text { Não conviver com pais biológicos; Viver com pai solteiro } \\
\text { ou família adotiva; Baixo nível de monitoramento } \\
\text { parental; Ter pelo menos } 2 \text { amigos do sexo masculino; } \\
\text { Sair com amigos depois da escola/à noite; Ter repetido } \\
\text { uma nota e não gosta da escola. }\end{array}$ & $\begin{array}{l}\text { Estimulação dos pais e da escola em } \\
\text { promover ações de saúde sexual positiva } \\
\text { na adolescência. }\end{array}$ \\
\hline $\begin{array}{l}\text { (Asubiaro; Fatusi, } \\
\text { 2014) } \\
\text { MEDLINE }\end{array}$ & $\begin{array}{l}\text { Transversal } \\
\text { Quantitativo } \\
6\end{array}$ & Desenvolvimento precoce da puberdade. & $\begin{array}{l}\text { Conhecimento sobre os riscos de } \\
\text { HIV/Aids; Religiosidade. }\end{array}$ \\
\hline $\begin{array}{l}\text { (Stephenson; Simon; } \\
\text { Finneran, 2014) } \\
\text { CINAHL }\end{array}$ & $\begin{array}{l}\text { Transversal } \\
\text { Quantitativo } \\
6\end{array}$ & $\begin{array}{l}\text { Conversar com pessoas fora do seio familiar; Uso de } \\
\text { bebida alcoólica; Trabalho precoce; Viver em comunidade } \\
\text { com mais adolescentes casados. }\end{array}$ & $\begin{array}{l}\text { Índice econômico elevado; Formação em } \\
\text { ensino secundário; Viver com os pais; } \\
\text { Monitorização parental elevada. }\end{array}$ \\
\hline $\begin{array}{l}\text { (Looze; Constantine; } \\
\text { Jerman; Vermeulen- } \\
\text { Smit; Bogt, 2015) } \\
\text { MEDLINE }\end{array}$ & $\begin{array}{l}\text { Transversal } \\
\text { Quantitativo } \\
6\end{array}$ & Pressão por parte dos amigos. & $\begin{array}{l}\text { Alta qualidade no relacionamento e uma } \\
\text { boa comunicação entre pais e } \\
\text { adolescentes. }\end{array}$ \\
\hline $\begin{array}{l}\text { Gonçalves et al., } \\
\text { 2015) } \\
\text { LILACS }\end{array}$ & $\begin{array}{l}\text { Descritivo } \\
\text { Quantitativo } \\
6\end{array}$ & $\begin{array}{l}\text { Baixo nível socioeconômico, Baixa escolaridade } \\
\text { associado a comportamento de risco, Usar bebida } \\
\text { alcoólica; Episódio de embriaguez; Experimentação de } \\
\text { fumo; Uso de drogas ilícitas; Envolvimento em brigas. }\end{array}$ & $\begin{array}{l}\text { Estratégias educativas para prevenção de } \\
\text { IST. }\end{array}$ \\
\hline $\begin{array}{l}\text { (Ortiz-Melgar; Pérez- } \\
\text { Saavedra; Valentín- } \\
\text { Ballarta; Zúñiga- } \\
\text { Hurtado, 2015) } \\
\text { LILACS }\end{array}$ & $\begin{array}{l}\text { Transversal } \\
\text { Quantitativo } \\
6\end{array}$ & Relação sexual ocasional; Consumo de álcool. & \\
\hline $\begin{array}{l}\text { (Okigbo, Kabiru, } \\
\text { Mumah, Mojola, } \\
\text { Beguy, 2015) } \\
\text { MEDLINE }\end{array}$ & $\begin{array}{l}\text { Coorte } \\
\text { Quantitativo } \\
4\end{array}$ & Não ter boa comunicação com a mãe. & $\begin{array}{l}\text { Boa comunicação e monitoramento } \\
\text { parental; Disciplina; Ações com inclusão } \\
\text { dos pais sobre saúde sexual e } \\
\text { reprodutiva. }\end{array}$ \\
\hline $\begin{array}{l}\text { (Espinosa- } \\
\text { Hernández; Bissel- } \\
\text { Havran; Nunn, 2015) } \\
\text { MEDLINE } \\
\end{array}$ & $\begin{array}{l}\text { Transversal } \\
\text { Quantitativo } \\
6\end{array}$ & $\begin{array}{l}\text { Religiosidade (religião católica) para adolescentes do sexo } \\
\text { masculino. }\end{array}$ & 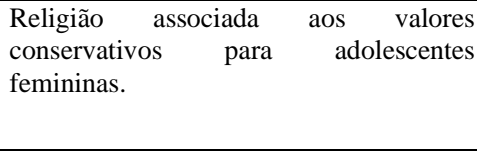 \\
\hline $\begin{array}{l}\text { (White; Warner, 2015) } \\
\text { MEDLINE }\end{array}$ & $\begin{array}{l}\text { Longitudinal } \\
\text { Quantitativo } \\
6\end{array}$ & $\begin{array}{l}\text { Aprovação materna para atividade sexual; Aprovação de } \\
\text { atividade sexual autodeclarada; Delinquência; Abuso } \\
\text { Infantil; Uso de bebida alcóolica. }\end{array}$ & 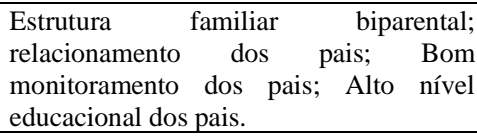 \\
\hline $\begin{array}{l}\text { (Heron et al., 2015) } \\
\text { MEDLINE }\end{array}$ & $\begin{array}{c}\text { Coorte } \\
\text { Quantitativo } \\
4\end{array}$ & $\begin{array}{l}\text { Residir em casa pobre; Baixa classe social; Baixa } \\
\text { escolaridade materna. }\end{array}$ & \\
\hline $\begin{array}{l}\text { (Homma; Wong; } \\
\text { Zumbo; Saewyc, 2015) } \\
\text { MEDLINE }\end{array}$ & $\begin{array}{l}\text { Transversal } \\
\text { Quantitativo } \\
6\end{array}$ & & $\begin{array}{l}\text { Maior comprometimento com grupos } \\
\text { étnicos; Maior nível de exploração da } \\
\text { identidade étnica; Maior exposição } \\
\text { cultural. }\end{array}$ \\
\hline
\end{tabular}




\begin{tabular}{|c|c|c|c|}
\hline $\begin{array}{l}\text { (Ayhan et al., 2015) } \\
\text { MEDLINE }\end{array}$ & $\begin{array}{l}\text { Ensaio clínico } \\
\text { multicêntrico } \\
\text { quantitativo } \\
6\end{array}$ & $\begin{array}{l}\text { Ser do sexo masculino; Primeira língua dos pais não ser o } \\
\text { francês; Consumo de bebida alcoólica; Consumo de } \\
\text { cannabis; Primeiro parceiro sexual mais velho. }\end{array}$ & $\begin{array}{l}\text { Religiosidade; Programa de intervenção } \\
\text { em ES para adolescentes escolares. }\end{array}$ \\
\hline $\begin{array}{l}\text { (Cheney, Oman; } \\
\text { Vesely; Aspy; Tolma; } \\
\text { John, 2015) } \\
\text { MEDLINE }\end{array}$ & $\begin{array}{l}\text { Coorte } \\
\text { Quantitativo } \\
4\end{array}$ & $\begin{array}{l}\text { Morte de familiares; Mudança nas finanças da família; } \\
\text { Conflitos familiares no domicílio; Doença da própria } \\
\text { pessoa; Fim das relações românticas. }\end{array}$ & Intervenções educativas. \\
\hline $\begin{array}{l}\text { (Markham et al., 2015) } \\
\text { MEDLINE }\end{array}$ & $\begin{array}{l}\text { Transversal } \\
\text { Quantitativo } \\
6\end{array}$ & Situações de risco; Uso de bebida alcóolica. & $\begin{array}{l}\text { Ações educativas na escola sobre } \\
\text { questões sexuais, situações de risco e uso } \\
\text { de substâncias. }\end{array}$ \\
\hline $\begin{array}{l}\text { (Decat et al., 2015) } \\
\text { MEDLINE }\end{array}$ & $\begin{array}{l}\text { Transversal } \\
\text { Quantitativo } \\
6\end{array}$ & $\begin{array}{l}\text { Idade mais avançada na adolescência; Não morar com os } \\
\text { pais; Ausência do convívio com a figura paterna; Residir } \\
\text { em bairros pobres; Uso de bebida alcóolica. }\end{array}$ & \\
\hline $\begin{array}{l}\text { (Skinner et al., 2015) } \\
\text { MEDLINE }\end{array}$ & $\begin{array}{c}\text { Coorte } \\
\text { Quantitativo } \\
4\end{array}$ & $\begin{array}{l}\text { Problemas comportamentais a partir dos } 5 \text { anos para } \\
\text { meninos; Problemas comportamentais a partir dos } 10 \text { anos } \\
\text { para meninas; Internalização dos problemas } \\
\text { comportamentais aos } 8 \text { e } 10 \text { anos de idade. }\end{array}$ & $\begin{array}{l}\text { Ações de promoção da saúde sexual } \\
\begin{array}{l}\text { considerando os } \\
\text { comportamento. }\end{array}\end{array}$ \\
\hline $\begin{array}{l}\text { Kastbom; Sydsjo; } \\
\text { Bladh; Priebe; Svedin, } \\
\text { 2015) } \\
\text { MEDLINE }\end{array}$ & $\begin{array}{l}\text { Transversal } \\
\text { Quantitativo } \\
6\end{array}$ & $\begin{array}{l}\text { Desemprego materno; Desatenção dos pais; Não morar } \\
\text { com os pais; Ser homossexual ou bissexual; Uso de } \\
\text { bebida alcoólica, fumar, drogas ilícitas e esteroides } \\
\text { anabolizantes; Comportamento antissocial; Baixa } \\
\text { autoestima; Ansiedade, Depressão; Abuso físico ou } \\
\text { sexual; Assistir pornografia. }\end{array}$ & \\
\hline $\begin{array}{l}\text { (González; Molina; } \\
\text { Luttges, 2015) } \\
\text { LILACS }\end{array}$ & $\begin{array}{l}\text { Transversal } \\
\text { Quantitativo } \\
6\end{array}$ & $\begin{array}{l}\text { Não ser estudante; Objetivo em terminar apenas o ensino } \\
\text { médio. }\end{array}$ & $\begin{array}{l}\text { Ter a escola como a principal fonte de } \\
\text { informações. }\end{array}$ \\
\hline $\begin{array}{l}\text { (Peltzer; Pengpid, } \\
\text { 2015) } \\
\text { WEB OF SCIENCE }\end{array}$ & $\begin{array}{l}\text { Transversal } \\
\text { Quantitativo } \\
6\end{array}$ & $\begin{array}{l}\text { Sexo masculino; Uso de fumo; Uso de bebida alcóolica; } \\
\text { Está em luta física nos últimos } 12 \text { meses; Comportamento } \\
\text { sedentário; Evasão escolar; Sentimento de ingratidão; } \\
\text { Falta de apego parental. }\end{array}$ & $\begin{array}{l}\text { Ações de programas de promoção à } \\
\text { saúde sexual abordando comportamentos } \\
\text { problemáticos como o uso de } \\
\text { substâncias, violência e evasão escolar. }\end{array}$ \\
\hline $\begin{array}{l}\text { (Avelar E Silva et al., } \\
\text { 2016) } \\
\text { MEDLINE }\end{array}$ & $\begin{array}{l}\text { Longitudinal } \\
\text { Quantitativo } \\
6\end{array}$ & $\begin{array}{l}\text { Não viver com pais biológicos; Baixa escolaridade; Uso } \\
\text { de bebida alcoólica e fumo; Usar TV em até 2horas/dia; } \\
\text { Uso de computadores em até } 2 \text { horas/dia. }\end{array}$ & \\
\hline $\begin{array}{l}\text { (Heywood et al., 2016) } \\
\text { MEDLINE }\end{array}$ & $\begin{array}{l}\text { Transversal } \\
\text { Quantitativo } \\
6\end{array}$ & Pressão dos amigos. & $\begin{array}{l}\text { Bom monitoramento parental; } \\
\text { Religiosidade e Cultura; Espera pelo } \\
\text { casamento; Medo de gravidez, IST/HIV; } \\
\text { Ações educativas promotoras de reforço } \\
\text { positivo para práticas sexuais. }\end{array}$ \\
\hline $\begin{array}{l}\text { (Johnson-Baker et al., } \\
\text { 2016) } \\
\text { MEDLINE }\end{array}$ & $\begin{array}{l}\text { Ensaio Clínico } \\
\text { Randomizado } \\
\text { Quantitativo } \\
2 \\
\end{array}$ & Ouvir quantidades elevadas de música rap. & \\
\hline $\begin{array}{l}\text { (Zito; Coster, 2016) } \\
\text { MEDLINE }\end{array}$ & $\begin{array}{l}\text { Quantitativo } \\
6\end{array}$ & 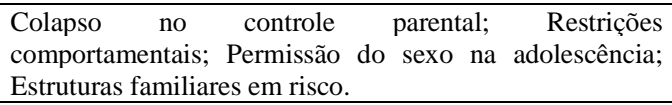 & \\
\hline $\begin{array}{l}\text { (Biney; } \quad \text { Dodoo, } \\
\text { 2016) } \\
\text { MEDLINE }\end{array}$ & $\begin{array}{l}\text { Quantitativo } \\
6\end{array}$ & $\begin{array}{l}\text { Assistir filmes de conteúdo pornográficos; Pertencer a } \\
\text { famílias de classe socioeconômica baixa. }\end{array}$ & \\
\hline $\begin{array}{l}\text { (Vandenbosch; } \\
\text { Beyens; Vangeel; } \\
\text { Eggermont, 2016) } \\
\text { MEDLINE } \\
\end{array}$ & $\begin{array}{l}\text { Longitudinal } \\
\text { Quantitativo } \\
\quad 6\end{array}$ & $\begin{array}{l}\text { Uso salas de bate-papo; Uso frequente de sites de } \\
\text { relacionamento; Uso frequente de site de conteúdos } \\
\text { pornográficos. }\end{array}$ & \\
\hline $\begin{array}{l}\text { (Rivera-Rivera et al., } \\
\text { 2016) } \\
\text { MEDLINE }\end{array}$ & $\begin{array}{l}\text { Transversal } \\
\text { Quantitativo } \\
6\end{array}$ & Crenças tradicionais; Alta autoestima social; Depressão. & $\begin{array}{l}\text { Ações educativas para promoção da } \\
\text { saúde sexual na perspectiva de gênero; } \\
\text { Boa comunicação entre pais e filhos. }\end{array}$ \\
\hline $\begin{array}{l}\text { (Avelar e Silva et al., } \\
\text { 2016) } \\
\text { MEDLINE }\end{array}$ & $\begin{array}{l}\text { Quantitativo } \\
6\end{array}$ & $\begin{array}{l}\text { Menor controle parental; Baixa qualidade no } \\
\text { relacionamento entre mães e pais. }\end{array}$ & Boa relação com a mãe. \\
\hline $\begin{array}{l}\text { (Spinola; Béria; } \\
\text { Schermann, 2017) } \\
\text { LILACS }\end{array}$ & $\begin{array}{l}\text { Transversal } \\
\text { Quantitativo } \\
6\end{array}$ & Menarca até 12 anos de idade; Parceiros Casuais. & $\begin{array}{l}\text { Papel do enfermeiro nas escolas, } \\
\text { planejamento e ações educativas em } \\
\text { saúde sexual e reprodutiva, por meio de } \\
\text { oficinas, envolvendo docentes, discentes, } \\
\text { pais e as lideranças da comunidade. }\end{array}$ \\
\hline $\begin{array}{l}\text { (Lowry; Robin; } \\
\text { Kann, 2017) } \\
\text { MEDLINE }\end{array}$ & $\begin{array}{c}\text { Transversal } \\
\text { Quantitativo } \\
6 \\
\end{array}$ & Relação sexual forçada; Violência; Abuso de substâncias. & \\
\hline
\end{tabular}


Research, Society and Development, v. 10, n. 14, e504101422505, 2021

(CC BY 4.0) | ISSN 2525-3409 | DOI: http://dx.doi.org/10.33448/rsd-v10i14.22505

\begin{tabular}{|c|c|c|c|}
\hline $\begin{array}{l}\text { (Doran; Waldron, } \\
\text { 2017) } \\
\text { MEDLINE }\end{array}$ & $\begin{array}{l}\text { Longitudinal } \\
\text { Quantitativo } \\
6\end{array}$ & Consumo de bebidas alcóolicas. & $\begin{array}{l}\text { Bom controle individual e familiar; } \\
\text { Ações promotoras de saúde com } \\
\text { abordagem multissistêmicas. }\end{array}$ \\
\hline $\begin{array}{l}\text { (Cabral et al., 2017) } \\
\text { MEDLINE }\end{array}$ & $\begin{array}{l}\text { Coorte } \\
\text { Quantitativo } \\
4\end{array}$ & $\begin{array}{l}\text { Pouco envolvimento parental; Boa interação social; } \\
\text { Imposição dos pares. }\end{array}$ & \\
\hline $\begin{array}{l}\text { (Gambadauro et al., } \\
\text { 2018) } \\
\text { MEDLINE }\end{array}$ & $\begin{array}{l}\text { Coorte } \\
\text { Quantitativo } \\
4\end{array}$ & $\begin{array}{l}\text { Uso de tabaco; Uso excessivo de bebida alcoólica; } \\
\text { Bullying; Exposição á brigas; Sono reduzido; Evasão } \\
\text { escolar; Baixo envolvimento com os pais. }\end{array}$ & \\
\hline $\begin{array}{l}\text { (Young; Burke; Nic } \\
\text { Gabhainn, 2018) } \\
\text { MEDLINE }\end{array}$ & $\begin{array}{l}\text { Quantitativo } \\
\quad 6\end{array}$ & $\begin{array}{l}\text { Residentes em bairros pobres ou em comunidades de } \\
\text { viajantes; Uso de bebida alcoólica, tabaco ou maconha } \\
\text { nos últimos } 30 \text { dias; Utilização de medicações para dores } \\
\text { de cabeça e de estômago; Bullying. }\end{array}$ & $\begin{array}{l}\text { Viver com ambos os pais; Maior } \\
\text { envolvimento com música ou teatro. }\end{array}$ \\
\hline $\begin{array}{l}\text { (Dufur; Hoffmann; } \\
\text { Erickson, 2018) } \\
\text { CINAHL }\end{array}$ & $\begin{array}{l}\text { Longitudinal } \\
\text { Quantitativo } \\
6\end{array}$ & Crianças criadas por pais solteiros. & \\
\hline $\begin{array}{l}\text { (Burke; Gabhainn; } \\
\text { Kelly, 2018) } \\
\text { WEB OF SCIENCE }\end{array}$ & $\begin{array}{l}\text { Transversal } \\
\text { Quantitativo } \\
6\end{array}$ & $\begin{array}{l}\text { Baixa classe social; amigos do sexo masculino; Pouco } \\
\text { apoio familiar; Comportamento de risco; Uso de bebida } \\
\text { alcóolica. }\end{array}$ & \\
\hline
\end{tabular}

Fonte: Elaborado pelos autores, Recife - PE (2018).

A análise do corpus textual, foi composta por 893 Segmentos de Textos (ST), relacionando 3.384 palavras que apresentaram ocorrência de 32058 vezes, subsidiando a produção do dendograma da Classificação Hierárquica Descendente (CHD), que reteve $83,20 \%$ do total de ST, dando origem à 3 classes (Figura 2). Cada classe foi representada pelas palavras mais significativas por meio do X2 e p-valor $(<0,05)$ com suas associações de acordo com as classes.

Figura 2. Dendograma da Classificação Hierárquica Descendente do corpus sobre "Fatores relacionados à iniciação das práticas sexuais precoces na adolescência”, Recife, PE, Brasil, 2018.

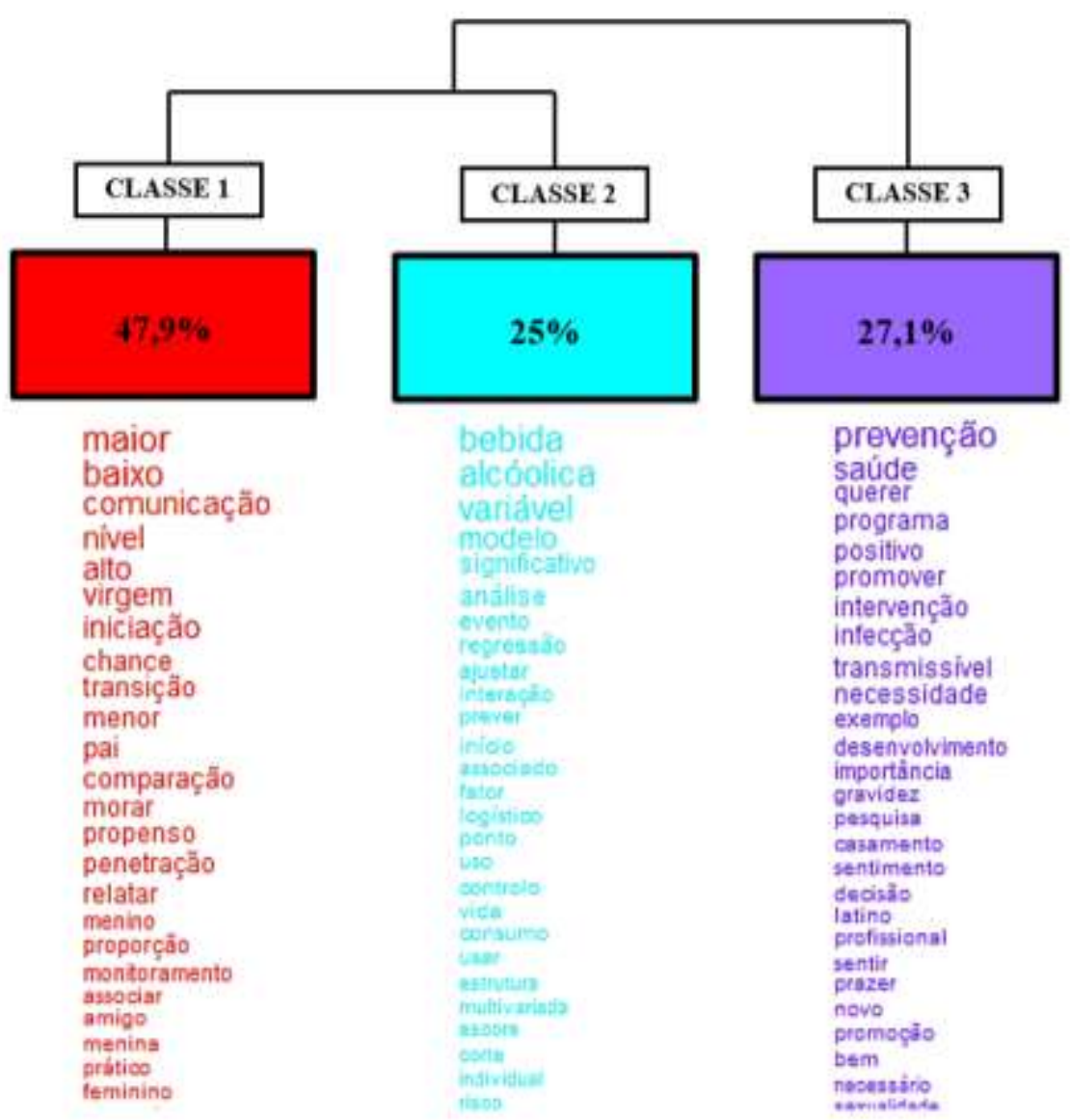

Fonte: Elaborado pelos autores. Adaptado do software IRAMUTEQ. Recife (2018). 
O dendograma possibilitou o reconhecimento das palavras mais significativas referentes aos ST associados a cada uma das classes, seguindo a ordem de participação e de proporção que representam a relação entre o corpus de forma totalizada. A delimitação em três classes decorreu em função da ocorrência e co-ocorrência das palavras com maior relevância. A classe 1 contribuiu com 47,9\% do total de ST e foi nomeada como "Vinculações familiares e entre os pares". A classe 2, nomeada "Situações de vulnerabilidade", conteve 25\% do total de ST. Por fim, a classe 3, denominada "Ações Promotoras de Saúde" representou $27,1 \%$ do total de ST.

Neste estudo, os artigos elegidos expõem e interpretam os fatores determinantes e protetores para iniciação das práticas sexuais precoces na adolescência. Foi possível perceber que diversos fatores associados à ISP, e que a depender do modo como é vivenciado pode apresentar efeitos positivos e negativos para o contexto familiar, social e intrínseco do adolescente, dificultando ou facilitando a abordagem de temas como esse nos ambientes, aos quais o público jovem costuma ter acesso. Estes fatores, por sua vez, são exibidos e discutidos conforme as classes identificadas pela CHD.

\section{Discussão}

Na classe 1 (Vinculações familiares e entre os pares) da CHD, foi observado uma influência das características sócio demográficas, econômicas e culturais que permeiam as relações familiares e sociais, responsáveis por determinar questões intersubjetivas e relacionais referentes ao comportamento sexual dos adolescentes.

A variável, gênero, constituiu um fator determinante considerando que há especificidades entre os motivos que concorrem para a iniciação sexual entre meninos e meninas (Ayhan et al., 2015; Peltzer; Pengpid, 2015). Para os adolescentes masculinos é requerido mostrar-se mais viril e mais másculo, por esse motivo eles são estimulados a iniciarem as práticas sexuais mais cedo do que as meninas (Silva et al., 2015).

Estudos destacaram as influências culturais como fatores associados à iniciação sexual dos adolescentes (Useche; Medina; Ross; Markham, 2014; Bámaca-Colbert; Greene; Killoren; Noah, 2014; Jovic et al., 2014; Homma; Wong; Zumbo; Saewyc, 2015; Heywood et al., 2016; Rivera-Rivera et al., 2016). Contudo, apenas dois estudos correlacionam a cultura como fator protetivo, capaz de adiar as práticas sexuais (Homma; Wong; Zumbo; Saewyc, 2015; Heywood et al., 2016). Embora a cultura pareça desempenhar um papel na diferenciação sexual entre os gêneros, sabe-se que a mesma é considerada heterogênea e dinâmica, podendo encorajar ou não, a ISP. Os costumes aos quais os adolescentes encontram-se inseridos, tendem a exercer modelos positivos para aquisição transgeracional identitária de um povo ou grupo populacional (Homma; Wong; Zumbo; Saewyc, 2015).

Emerge considerar que os costumes apreendidos pelos adolescentes sofrem influência de modelos hegemônicos. Desse modo, o comportamento do adolescente em relação ao seu status sexual apresenta relação direta com a construção social/cultural, que passa a impregnar as relações intra e extra familiar, uma vez que é exercida uma maior cobrança de conduta comportamental para manter a virgindade sobre as meninas e maior liberdade de iniciação das práticas sexuais para os meninos (Tenório et al., 2016). Estudo desenvolvido no Quênia aferiu que o monitoramento parental, a disciplina e a comunicação entre pais e adolescentes, predizem um atraso na transição para a primeira relação sexual apenas para adolescentes do sexo feminino (Okigbo et al., 2015).

Ao considerar os aspectos socioeconômicos, estudos desenvolvidos no Brasil, Reino Unido, Nicarágua, Gana e Irlanda evidenciaram que os adolescentes pertencentes a famílias de classes sociais desfavorecidas e residentes em bairros com alto índice de pobreza apresentam maior vulnerabilidade a iniciar precocemente as práticas sexuais (Gonçalves et al., 2015; Heron et al., 2015; Decat et al., 2015; Biney; Dodoo, 2016; Young; Burke; Nic Gabhainn, 2018; Burke; Gabhainn; Kelly, 2018). Em um estudo usando dados longitudinais de transições juvenis de saúde sexual e reprodutiva na Nigéria e no Quênia 
comprovou que a iniciação sexual e a primeira gravidez de jovens com maior nível de instrução educacional e maior poder aquisitivo é mais tardia do que nos seus colegas menos instruídos e mais pobres (Speizer et al., 2017).

Ainda sobre as vinculações familiares os estudos ressaltaram, que não conviver com pais biológicos (Useche; Medina; Ross; Markham, 2014; Jovic et al., 2014; Avelar e Silva et al., 2016); viver com pai solteiro (Jovic et al., 2014); baixo nível de monitoramento/controle parental (Jovic et al., 2014; Kastbom et al., 2015; Zito, Coster, 2016; Avelar \& Silva et al., 2016, Cabral et al., 2017; Gambadauro et al., 2018); instabilidade familiar (namoro/materno) e atividade permissiva (Zito, Coster, 2016); conflitos familiares no ambiente domiciliar (Markham et al., 2015); superproteção (Kastbom et al., 2015); pouco apoio familiar (Burke; Gabhainn; Kelly, 2018) e comunicação sobre sexo fora do seio familiar (Stephenson; Simon; Finneran, 2014) são fatores determinantes para a iniciação das práticas sexuais precoces na adolescência.

Estudos reforçaram a existência de fatores protetores capazes de adiar a IS sexuais nesta população, são eles: vivência com os pais (Stephenson; Simon; Finneran, 2014; Young; Burke; Nic Gabhainn, 2018), níveis elevados de monitorização parental (Stephenson; Simon; Finneran, 2014; Doran; Waldron, 2017), boa comunicação com a mãe (Okigbo et al., 2015; Avelar \& Silva et al., 2016) e pressão dos pais relacionadas as adolescentes do sexo feminino (Heywood et al., 2016).

Uma relação dialógica e de confiança, entre pais e adolescentes contribuem para a formação da personalidade do indivíduo, sendo capaz de auxiliar na preparação para a fase adulta, através dos valores e modelos apreendidos. Neste sentido, o fortalecimento das relações parentais é capaz de enfatizar de forma positiva o direcionamento comportamental para postergar a iniciação das atividades sexuais de adolescentes (Oliveira; Leite Junior; Nascimento, 2017; Febrasgo, 2017).

Estudos qualitativos no Brasil observaram que ainda há um tabu em abordar sobre sexualidade no ambiente familiar. Contudo, na existência de diálogo, essa temática foi relacionada apenas às questões biológicas ligadas ao coito, contracepção, e prevenção de IST, prevalecendo as restrições/proibições relacionadas à iniciação da vida sexual do adolescente (Savegnago; Arpini, 2016; Nery et al., 2015).

Na relação entre os pares, o ambiente escolar emergiu como um poderoso locus capaz de provocar interferências na vida do adolescente, sendo possível perceber que as relações escolares necessitam apresentar um equilíbrio entre o adiamento das relações sexuais precoces e a ausência de limites impostos aos adolescentes. Com base nesses aspectos, foram vistas questões relacionadas à evasão escolar (Pelzer; Pengpid, 2015; Gambadauro et al., 2018); baixa escolaridade (Avelar e Silva et al., 2016); não ser estudante (González; Molina; Luttges, 2015); insegurança no ambiente escolar (Jeremic; Matejic; Soldatovic; Radenovic, 2014); insatisfação com a escola (Jovic et al., 2014); não apresentar bons rendimentos escolares (Jeremic; Matejic; Soldatovic; Radenovic, 2014; Jovic et al., 2014); apenas terminar o ensino médio (González; Molina; Luttges, 2015); e aprovação dos pares no contexto escolar (White; Warner, 2015) sendo considerados fatores determinantes para iniciação das práticas sexuais precoces na adolescência.

Em relação às vinculações de pares, estudos realizados na França, Holanda, Austrália e Irlanda referem que a estimulação sexual entre os adolescentes emerge da difusão de imposições sociais que moldam os comportamentos dos mesmos, desprovidos de uma conscientização e de valores éticos e morais (Jovic et al., 2014; Looze et al., 2015; Heywood et al., 2016; Young; Burke; Nic Gabhainn, 2018; Burke; Gabhainn; Kelly, 2018). Corroborando com os achados da literatura, no estudo foi identificado que ter amigos do sexo masculino, boa comunicação, pressão por parte dos amigos e sair com eles frequentemente depois da escola ou à noite potencializam a iniciação das práticas sexuais precocemente.

A precocidade da atividade sexual dos adolescentes, também é influenciada por fatores relacionados ao contexto escolar, como na reprovação do ano letivo e na evasão escolar (Santos et al., 2018). Essa conjuntura apresenta um caráter preocupante, uma vez que as reprovações estudantis, não se limitam a repercussões no âmbito acadêmico, podendo ocasionar transtornos nos aspectos sociais, com a exposição a riscos para a saúde. 
A escola deve ser vista como um lugar privilegiado para discutir de forma dialógica e igualitária temáticas sobre sexualidade na adolescência, vislumbrando mudanças positivas para o comportamento sexual seguro na adolescência (Moraes; Brêtas; Vitalle, 2018). A permanência no ambiente escolar e a formação do ensino secundário foram elencados como fatores protetores (Stephenson; Simon; Finneran, 2014). A escola foi reconhecida como principal fonte de informação e de ES (González; Molina; Luttges, 2015). Quando as escolas são subutilizadas, há substituição pelas informações dos pares e pelos conteúdos expostos nos ambientes virtuais, considerados muitas vezes inapropriados, por ampliar a exposição dos adolescentes a situações de vulnerabilidade (Lara; Abdo, 2016).

A classe 2 da CHD (Situações de vulnerabilidade) faz alusão às questões de vulnerabilidade vivenciadas pelos adolescentes em seu convívio social e que apresentam interferência na iniciação das atividades sexuais precoces. As vulnerabilidades apresentadas nos estudos envolveram principalmente: consumo de bebida alcoólica (Jeremic; Matejic; Soldatovic; Radenovic, 2014; Stephenson; Simon; Finneran, 2014; Gonçalves et al., 2015; Ortiz-Melgar et al., 2015; Ayhan et al., 2015; Markhan et al., 2015; Decat et al., 2015; Kastbom et al., 2015; Peltzer; Pengpid, 2015; Avelar \& Silva et al., 2016; Doran; Waldron, 2017; Oliveira, Leite Junior; Nascimento, 2017); uso de tabaco (Jeremic; Matejic; Soldatovic; Radenovic, 2014; Gonçalves et al., 2015; Kastbom et al., 2015; Peltzer; Pengpid, 2015; Avelar \& Silva et al., 2016; Young; Burke; Nic Gabhainn, 2018) e utilização de drogas ilícitas (Jeremic; Matejic; Soldatovic; Radenovic, 2014; Gonçalves et al., 2015; Ayhan et al., 2015; Kastbom et al., 2015; Lowry; Robin; Kann, 2017; Gambadauro et al., 2018; Young; Burke; Nic Gabhainn, 2018).

Os adolescentes reconhecem a ingestão de bebida alcoólica como um meio capaz de induzir ao ato sexual, transformando esse momento em relações afetivas superficiais e nem sempre consensuais, com repercussão negativa no desenvolvimento da sexualidade. O uso de álcool antes ou durante as relações sexuais é reforçado por uma crença subjetiva de que pode servir como um dinamizador dos sentimentos de medo e angústia, ao diminuir a timidez e facilitar a performance sexual e o prazer (Dallo; Martins, 2018).

Resultado semelhante foi encontrado no estudo realizado em Olinda/Pernambuco, que verificou uma associação significativa entre o consumo de bebida alcoólica nos últimos 30 dias e já ter tido relações sexuais. Essa associação pode ser decorrente do efeito desinibitório que a substância alcóolica proporciona e que acaba por facilitar a relação sexual precoce. Constitui um mito acreditar que o uso de álcool aumenta a libido e melhora o desempenho sexual, uma vez que o mesmo reage interferindo o juízo crítico do adolescente na tomada de decisão para utilização segura do preservativo nas relações sexuais (Costa et al., 2017).

Ao destacar a existência de elevadas taxas de consumo de álcool e drogas entre os adolescentes, estudo brasileiro constatou uma associação entre o uso de drogas lícitas e ilícitas com o gênero dos adolescentes, de forma que os meninos relataram níveis de consumo mais elevados do que as meninas. Esse comportamento de risco dos meninos apresenta uma correlação com a falta de moradia e abandono familiar. É válido salientar que meninos que vivem em condições de adversidade social estão mais propensos a se envolver com o uso de álcool e drogas ilícitas e consequentemente estarem mais expostos a se engajar em atividades sexuais cada vez mais precoces (Penelope Morrison; Smith; Akers, 2014).

Autores relatam que o tabagismo vem sendo iniciado precocemente, quase sempre no período da adolescência sendo responsável por altas taxas de morbimortalidade em todo o mundo (Jeremic; Matejic; Soldatovic; Radenovic, 2014; Gonçalves et al., 2015; Kastbom et al., 2015; Peltzer; Pengpid, 2015; Avelar \& Silva et al., 2016; Young; Burke; Nic Gabhainn, 2018). Em associação do tabagismo aos aspectos sexuais na adolescência, estudo com 370 adolescentes na faixa etária de 10 à 19 anos apresentou que o consumo tinha correlação com o início precoce das práticas sexuais nessa população, apresentando uma maior vulnerabilidade em indivíduos do sexo masculino (Viana et al., 2018; Malta et al., 2014).

A classe 3 da CHD (Ações Promotoras de Saúde) faz referência às ações de saúde sexual e reprodutiva para o público adolescente, propondo intervenções educativas como ferramentas eficazes para trabalhar temáticas sobre sexualidade. Estudos 
mostram os benefícios das intervenções de educação em saúde sexual para população adolescente, como retardamento da IS, redução dos comportamentos de riscos, prevenção de gravidez na adolescência e de IST/Aids (Jeremic; Matejic; Soldatovic; Radenovic, 2014; Jovic et al., 2014; Asubiaro; Fatusi, 2014; Gonçalves et al., 2015; Spinola; Béria; Schermann, 2017; Espinosa-Hernández; Bissel-Havran; Nunn, 2015; White; Warner, 2015; Cheney et al., 2015; Markham et al., 2015; Doran; Waldron, 2017; Ayhan et al., 2015; Skinner at al., 2015; Heywood et al., 2016; Peltzer; Pengpid; 2015; Rivera-Rivera et al., 2016).

Estudos recomendam a utilização de intervenções educativas na perspectiva de uma abordagem holística acerca das necessidades sexuais desses indivíduos, ao considerar o adolescente e o ambiente ao qual o mesmo encontra-se inserido como parte da complexidade do seu ser (Doran; Waldron, 2017; Young; Burke; Nic Gabhainn, 2018). Neste contexto, é necessária uma reorganização das políticas públicas de saúde para as práticas de promoção da ES, incluindo a perspectiva de gênero para fortalecer a autoestima dos adolescentes e seu poder de raciocínio crítico para lidar com as adversidades, além de estimular a comunicação entre pais e filhos no intuito de adiar as práticas sexuais (Rivera-Rivera et al., 2016).

Destarte que a ES deve fortalecer o público adolescente para tomada de decisões seguras em relação a sua iniciação sexual, sendo necessário desnudar a temática sexualidade não apenas no âmbito biológico, mas também promovendo conhecimento, reflexão autônoma e enfatizando o prazer, os sentimentos, o respeito e a responsabilidade dos envolvidos (Sfair; Bittar; Lopes, 2015). Neste cenário, destaca-se o ambiente escolar como um espaço para realização de ações de ES com os adolescentes, visto que, os mesmos passam a maior parte do tempo na escola e sentem-se confiantes para discutir os assuntos relacionados à sexualidade, pois estão no grupo de iguais com o qual apresentam maior afinidade (Kauffman; Luz, 2020).

Revisão sistemática evidenciou que apesar das práticas de ES não serem recentes, ainda existe a necessidade de avanços na área da ES nas escolas brasileiras voltadas a prevenção da saúde do adolescente, exibindo que as ações ainda estão pautadas em um tratamento moral e pedagógico. Constitui um desafio a realização de ações interdisciplinares de educação em saúde sexual que abordem a temática no contexto escolar, atendendo as demandas e expectativas dos adolescentes, com vistas à superação das barreiras que impedem a consolidação de práticas educativas que ampliam o acesso aos adolescentes, genitores, profissionais da saúde e professores (Furlanetto et al., 2018).

Em apenas três estudos os autores recomendaram a participação efetiva dos genitores frente às intervenções promotoras e educativas de saúde sexual, no intuito de fortalecer os cuidados dos adolescentes vislumbrando um adiamento das práticas sexuais nesses indivíduos (White; Warner, 2015; Rivera-Rivera et al., 2016; Spinola; Béria; Schermann, 2017). Ademais, um estudo realizado nas Bahamas destacou que a exposição apenas dos jovens a ações de intervenções educativas e promotoras de saúde sexual não melhora o conhecimento dos pais, ressaltando que a participação dos genitores amplia as possibilidades de diálogo entre pais/filhos sobre as questões mais complexas e de forma qualificada acerca da iniciação sexual na adolescência e a redução dos riscos sexuais (Dinay-Koci et al., 2015).

O papel dos profissionais de saúde enquanto educadores é expresso em estudo que destaca a importância de uma ação intersetorial nas escolas, realizando atividades de planejamento e trabalhos educacionais na perspectiva da saúde sexual e reprodutiva, utilizando oficinas lúdicas, capazes de envolver docentes, discentes, pais e as lideranças comunitárias (Spinola; Béria; Schermann, 2017). Destarte a necessidade de fortalecer uma comunicação e cooperação entre os profissionais da rede de serviços de saúde e construir uma estrutura de apoio ao sistema público de saúde nas ações de promoção à saúde da população adolescente (Almeida; Fausto; Giovanella, 2011).

O profissional de saúde exerce o cuidado enquanto educa, e, para tanto, deverá ter conhecimento das novas possibilidades educativas agregando a prática assistencial evidências científicas (Farre et al., 2018). Emerge destacar que a ES dos adolescentes precisa ser realizada antes IS, auxiliando o adolescente a compreender as modificações e o desenvolvimento do seu corpo, no intuito de promover atitudes e relacionamentos responsáveis (Spinola; Béria; Schermann, 2017). 


\section{Considerações Finais}

Observou-se que os fatores associados a uma sexarca precoce entre os adolescentes têm sido amplamente discutidos no meio científico, porém a abordagem da temática com esse público ainda é restritiva as consequências e riscos que envolvem as práticas sexuais, e não considera os fatores que determinam sua ocorrência, deixando lacunas a serem discutidas no âmbito amplo da saúde sexual e reprodutiva em uma abordagem promotora de saúde.

Uma diversidade de fatores foram relacionados à ISP na adolescência, sendo apreciados a partir do contexto familiar, escolar, situações de vulnerabilidade e ações promotoras de saúde. No contexto familiar constituíram fatores determinantes a ISP, ser adolescentes de famílias com classe social desfavorecida; ausência de diálogo entre pais e adolescentes; não conviver com pais biológicos; baixo nível de monitoramento dos pais; instabilidade e conflitos no ambiente familiar. Entretanto, foi possível constatar que os adolescentes do sexo masculino apresentam uma maior vulnerabilidade para uma sexarca cada vez mais precoce em comparação as adolescentes do sexo feminino, influenciados por uma pressão social que impõe um comportamento de virilidade.

No enfrentamento da exposição do adolescente a uma ISP, foram evidenciadas como medidas protetivas a sedimentação de conhecimentos consistentes sobre sexualidade e prática sexual no ambiente familiar, requerendo o desenvolvimento de uma relação de empatia e confiança entre pais e filhos, para tratar da temática.

No âmbito escolar emergiu como fatores relacionados à precocidade das práticas sexuais a evasão escolar; baixa escolaridade; insatisfação com a escola; baixo rendimento escolar; não ser estudante; ter amigos do sexo masculino; sofrer influência ou pressão dos amigos e sair com eles frequentemente depois da escola ou à noite. Como fatores protetivos, neste cenário, destacam-se a formação em ensino secundário, ter o ambiente escolar como principal fonte de informação e a realização de ações de promoção à saúde na escola.

Em relação às situações de vulnerabilidade foi verificado uma associação entre o consumo abusivo de bebidas alcóolicas e drogas ilícitas, com a ocorrência de sexarca precoce na adolescência, concorrendo para a exposição de comportamentos promíscuos relacionados às práticas de sexo casual.

O entendimento amplo de saúde não pode desconsiderar as questões de sexualidade e a precocidade das práticas sexuais na população adolescente, que podem desencadear repercussões bio psico sócio culturais prejudiciais, quando os adolescentes não dispõem de uma rede de apoio voltada à saúde integral.

A educação em saúde constitui uma estratégia promotora de saúde do adolescente, ao propor a construção de conhecimentos interdisciplinares capazes de articular o pensamento crítico e as emoções ao lidar com situações que possam trazer consequências à sua saúde no futuro. Cabe aos profissionais de saúde articular junto à gestão escolar, ações educativas em saúde integradas ao projeto pedagógico, que favoreça a saúde sexual e reprodutiva do adolescente em consonância com uma formação cidadã e com o seu empoderamento para a tomada de decisão, em um contexto de respeito e apoio a diversidade de gênero e orientação sexual.

As limitações do presente estudo estão relacionadas ao número elevado de estudos primários elegíveis para compor a revisão integrativa da literatura, evidenciando a complexidade e multifatorialidade que concorrem para a ISP, como também, atrelando este problema de saúde pública a outras situações de vulnerabilidade; demandando um recorte temporal mais restrito para maior profundidade nas discussões. Cabe ainda destacar um número mais reduzido de estudos que apreciam os fatores protetivos em relação aos fatores de vulnerabilidade para a iniciação precoce na atividade sexual. 


\section{Referências}

Almeida, P. F., Fausto, M. C. R., \& Giovanella, L. (2011). Fortalecimento da atenção primária à saúde: estratégia para potencializar a coordenação dos cuidados. Rev Panam Salud Publica. 29(2), 84-95.

Alves, J. S. A., et al. (2021). Socioeconomic characteristics influence attitudes towards sexuality in adolescents. J Hum Growth Dev. 31(1), 101-115.

Aromataris, E., \& Munn, Z. (2017). Joanna Briggs Institute Reviewer's Manual. The Joanna Briggs Institute.

Asubiaro, O. Y., \& Fatusi, A. O. (2014). Differential effects of religiosity on sexual initiation among Nigerian in-school adolescents. International Journal of Adolescent Medicine and Health, 26(1), 93-100.

Avelar e Silva., R. N., et al. (2016). Early Sexual Intercourse: Prospective Associations with Adolescents Physical Activity and Screen Time. PLoS One, 11(8), e0158648.

Avelar e Silva, R. N., et al. (2016). Mother- and Father-Adolescent Relationships and Early Sexual Intercourse. Pediatrics. 138(6), :1-9.

Ayhan, G., et al. (2015). Prevalence and risk factors of early onset of sexual intercourse in a random sample of a multiethnic adolescent population in French Guiana. AIDS Care, 27(8), 1025-1030.

Bámaca-Colbert, M. Y., Greene, K. M., Killoren, S. E., \& Noah, A. J. (2014). Contextual and Developmental Predictors of Sexual Initiation Timing among Mexican-origin Girls. Developmental Psychology, 50(10), 2353-2359.

Barth, B., Wagner, A., \& Levandowski, D. C. (2017). Chronological description of south Brazilian adolescents romantic behaviors. Psicologia: Teoria e Prática, 19(3), 302-315.

Biney, A. A. E., \& Dodoo, F. N. A. (2016). What are the characteristics of 'sexually ready' adolescents? Exploring the sexual readiness of youth in urban poor Accra. BMC Public Health, 16(9), 1-11.

Burke, L., Gabhainn, S. N., \& Kelly, C. (2018). Socio-Demographic, Health and Lifestyle Factors Influencing Age of Sexual Initiation among Adolescents. Int J Environ Res Public Health. 15(9), 1-15.

Cabral, P., et al. (2017). Generational status and social factors predicting initiation of partnered sexual activity among latino/a youth. Health Psychol. 36(2), 169-78.

Campos, H. M., et al. (2017). Direitos humanos, cidadania sexual e promoção de saúde: diálogos de saberes entre pesquisadores e adolescentes. Saúde Debate. 41(113), 658-669.

Chaves, A. C. P., et al. (2014). Conhecimentos e atitudes de adolescentes de uma escola pública sobre a transmissão sexual do HIV. Rev. bras. enferm. 67(1), 48-53.

Cheney, M. K., et al. (2015). Prospective Association Between Negative Life Events and Initiation of Sexual Intercourse: The Influence of Family Structure and Family Income. American Public Health Association, 105(3), 598-604.

Costa, A. C. Q., et al. (2017). Álcool e comportamento sexual entre estudantes do ensino médio no Brasil. Adolesc. Saude. 14(3), 24-29.

Critical Appraisal Skills Programme [Internet] (2013). Oxford: CASP. https://casp-uk.net/casp-tools-checklists/

Dallo, L., \& Martins, R. A. (2018). Association between the risk of alcohol use and unprotected sex in adolescents in a city in the southern region of Brazil. Ciênc. saúde coletiva. 23(1), 303-314.

Decat, P., et al. (2015). Sexual onset and contraceptive use among adolescents from poor neighbourhoods in Managua, Nicaragua. The European Journal of Contraception \& Reproductive Health Care, 20(2), 88-100.

Dinay-Koci, V., et al. (2015). Adolescent Sexual Health Education: Parents Benefit Too. Health Educ Behav. 42(5),648-653.

Doran, K. A., \& Waldron, M. (2017). Timing of First Alcohol Use and First Sex in Male and Female Adolescents. J Adolesc Health. 61(5), 606-11.

Dufur, M. J., Hoffmann, J. P., \& Erickson, L. D. (2018). Single Parenthood and Adolescent Sexual Outcomes. Journal of Child and Family Studies. 27, 80215.

Espinosa-Hernández, G., Bissel-Havran, J., \& Nunn, A. (2015). The Role of Religiousness and Gender in Sexuality Among Mexican Adolescents. The Journal of Sex Research, 52(8), 887-897.

Faria, C. S., \& Martins, C. B. G. (2016). Violence among adolescent students: conditions of vulnerability. Enfermería global, 15(42), 185-198.

Farre, A. G. M. C., et al. (2018). Adolescent health promotion based on community-centered arts education. Rev. Bras. Enferm. 71(1), 26-33.

Federação Brasileira das Associações de Ginecologia e Obstetrícia - FEBRASGO. Sexualidade na adolescente - Série orientações e recomendações. São Paulo: Connexamm; 2017.

Furlanetto, M. F., et al. (2018). Sexual education in brazilian education: systematic revision of the literature. Cad. Pesqui. 48(168), 550-571.

Gambadauro, P., et al. (2018). Correlates of sexual initiation among European adolescents. PLoS One. 13(2), 1-16.

Genz, N., Meincke, S. M. K., Carret, M. L. V., Corrêa, A. C. L., \& Alves, C. N. (2017). Sexually transmitted diseases: knowledge and sexual behavior of adolescents. Texto \& Contexto Enfermagem, 26(2), e5100015. 
Gonçalves, H., et al. (2015). Sexual initiation among adolescents (10 to 14 years old) and health behaviors. Revista brasileira de epidemiologia, $18(1)$, 1-18.

González, A. E., Molina, G. T., \& Luttges, D. C. (2015). Características de la educación sexual escolar recibida y su asociación con la edad de inicio sexual y uso de anticonceptivos en adolescentes chilenas sexualmente activas. Revista chilena de obstetricia y ginecologia, 80(1), 24-32.

Heron, J., et al. (2015). Social Factors Associated with Readiness for Sexual Activity in Adolescents: A Population-Based Cohort Study. Archives of Sexual Behavior, 44(3), 669-678.

Heywood, W., et al. (2016). Mitchell A. "Dude, I'm Seventeen... It's Okay Not to Have Sex by This Age": Feelings, Reasons, Pressures, and Intentions Reported by Adolescents Who Have Not Had Sexual Intercourse. The Journal of Sex Research, 53(9), 1207-1214.

Homma, Y., Wong, S. T., Zumbo, B. D., \& Saewyc, E. M. (2015). Ethnic Identity and Sexual Initiation Among East Asian Youth in Canada. Journal of Immigrant and Minority Health, 17(5), 1580-1584.

Jeremic, V., Matejic, B., Soldatovic, I., \& Radenovic, S. (2014). Early sexual initiation and risk factors in Serbian adolescents: data from the National Health Survey. The European Journal of Contraception \& Reproductive Health Care, 19(3), 211-219.

Johnson-Baker, K. A., et al (2016). Rap Music Use, Perceived Peer Behavior, and Sexual Initiation Among Ethnic Minority Youth. Journal of Adolescent Health, 58(3), 317-322.

Jovic, B. S., et al. (2014). Associations Between Life Contexts and Early Sexual Initiation Among Young Women in France. Perspect Sex Reprod Health, 46(1), 31-39.

Kastbom, A. A., et al. (2015). Sexual debut before the age of 14 leads to poorer psychosocial health and risky behaviour in later life. Acta Paediatrica, 104(1), 91-100.

Lara, L. A. S., \& Abdo, C. H. N. (2016). Age at initial sexual intercourse and health of adolescent girls. J Pediatr Adolesc Gynecol. 29(5), 417-423.

Leci Kauffman, L., \& Luz, F. A. (2020). Sexualidade na sala de aula: visão de alunos do ensino fundamental no município de dom Pedrito -RS. Revista Diversidade e Educação. 8(1), 238-258.

Lino, C. R. M., et al. (2017). Adaptação transcultural de instrumentos de pesquisa conduzida pela enfermagem do brasil: Uma revisão integrativa. Texto \& Contexto - Enfermagem [online]. 26(4), e1730017.

Looze, M., et al. (2015). Parent-Adolescent Sexual Communication and Its Association With Adolescent Sexual Behaviors: A Nationally Representative Analysis in the Netherlands. The Journal of Sex Research, 52(3), 257-268.

Lowry, R., Robin, L., \& Kann, L. (2017). Effect of Forced Sexual Intercourse on Associations Between Early Sexual Debut and Other Health Risk Behaviors Among US High School Students*. J Sch Health. 87(6), 435-47.

Malta, D. C., et al. (2014). Exposure to alcohol among adolescent students and associated factors. Rev. Saúde Pública. 48(1), 52-62.

Marçal, V., \& Miranda, J. C. (2021). Desenvolvimento do jogo didático "Perfil - Educação Sexual" como ferramenta integrada ao ensino na educação básica. Arquivos do Mudi. 25(2), 27-48.

Markham, C. M., et al. (2015). Factors Associated With Early Sexual Experience Among American Indian and Alaska Native Youth. Journal of Adolescent Health, 57(3), 334-341.

Melnyk, B. M., Fineout-Overholi, E. (2011). Evidence -based practice in nursing \& healthcare: guide to best practice. (2 ${ }^{\mathrm{a}}$ ed.) New York: Lippincott Williams \& Wilkins.

Moraes, S. P., Brêtas, J. R. S., \& Vitalle, M. S. S. (2018). School Education, Sexuality and Adolescence: a Systematic Review. J Health Sci. 20(3), 221-230.

Nery, I. S., et al. (2015). Approach to sexuality in the dialogue between parents and adolescents. Acta paul. enferm. 28(3), 287-292.

Okigbo, C. C., et al. (2015). Influence of parental factors on adolescents' transition to first sexual intercourse in Nairobi, Kenya: a longitudinal study. Reprod Health, 12(73), 1-12.

Oliveira, P. W. L., Leite Junior, F. F., \& Nascimento, F. A. (2017). ADOLESCÊNCIA E A FAMÍLIA: Desafios para uma educação sexual dos/as filhos/as. Revista Café com Sociologia. 6(2), :229-249.

Ortiz-Melgar, M., et al. (2015). Asociación entre consumo de alcohol y relaciones sexuales ocasionales en los adolescentes. Revista Enfermeria Herediana, $8(2), 110-115$.

Peltzer, K., \& Pengpid, S. (2015). Early Sexual Debut and Associated Factors among In-school Adolescents in Six Caribbean Countries. West Indian Medical Journal. 64(4), 351-56.

Penelope Morrison, P., Smith, A. E., \& Akers, A. (2014). Substance use and sexual risk among at-risk adolescents in Juiz de Fora, Minas Gerais State, Brazil. Cad. Saúde Pública. 30(4), 794-804.

Rivera-Rivera, L., et al. (2016). Sexual intercourse debut and associated factors in Mexican students aged 14-19 years in public schools. Gac Sanit, 30(1), 2430 .

Santos, R. M., et al. (2018). School failure and health and social aspects: A cross-sectional study with adolescents. Rev baiana enferm. 32(e21827), 1-10. 
Research, Society and Development, v. 10, n. 14, e504101422505, 2021

(CC BY 4.0) | ISSN 2525-3409 | DOI: http://dx.doi.org/10.33448/rsd-v10i14.22505

Santos, M. P., Farre, A. G. M. C., Bispo, M. S., Sousa, L. B., \& Marinho, D. D. T. (2017). Promoting adolescente sexual and reproductive health: peer education. Revista Baiana de Enfermagem, 31(3), 1-8.

Savegnago, S. D. O., \& Arpini D. M. (2016). A Abordagem do tema sexualidade no contexto familiar: o ponto de vista de mães de adolescentes. Psicologia: Ciência e Profissão. 36(1), 130-144.

Sfair, S. C., Bittar, M., \& Lopes, R. E. (2015). Educação sexual para adolescentes e jovens: mapeando proposições oficiais. Saúde Soc. 24(2), 620-632.

Silva, A. S. N., et al. (2015). Início da vida sexual em adolescentes escolares: um estudo transversal sobre comportamento sexual de risco em Abaetetuba, Estado do Pará, Brasil. Rev Pan-Amaz Saude. 6(1), 27-34.

Skinner, S. R., et al. (2015). Childhood Behavior Problems and Age at First Sexual Intercourse: A Prospective Birth Cohort Study. Pediatrics, 35 (2), $255-263$.

Souza, M. A. R., et al. (2018). The use of IRAMUTEQ software for data analysis in qualitative research. Rev da Esc Enferm da USP. 52(e03353-e03353), 17.

Speizer, I. S., et al. (2017). Examination of youth sexual and reproductive health transitions in Nigeria and Kenya using longitudinal data. BMC Public Health. $17(1), 142$.

Spinola, M. C. R. (2020). Fatores associados à iniciação sexual precoce de adolescentes em Santarém-PA. SANARE (Sobral, Online). 19(1), 36-47.

Spinola, M. C. R., Béria, J. U., \& Schermann, L. B. (2017). Factors associated with first sexual intercourse among mothers with 14-16 years of age from Porto Alegre/RS, Brazil. Ciênc. saúde coletiva. 22(11), 3755-62.

Stephenson, R., Simon, C., \& Finneran, C. (2014). Community Factors Shaping Early Age at First Sex among Adolescents in Burkina Faso, Ghana, Malawi, and Uganda. Journal of Health, Population and Nutrition, 32(2), 161-175.

Tenório, T. S. (2016). Tabu e repressão: A sexualidade Feminina sob a ótica de Gabriela Masson, em Garota Siririca. Boletim Historiar. (14), $28-37$.

Torraco, R. J. (2016). Writing integrative literature reviews: using the past and present to explore the future. Hum Resour Dev Int. 15(4), 404-28.

Useche, B., Medina, G., Ross, M. W., \& Markham, C. (2014). Your Clothes Aren't Going to Magically Come Off': An Exploratory Study of U.S. Latino Adolescents' Reasons for Having or Not Having First-Time Vaginal Intercourse. Universitas Psychologica, 13(4), 1409-1418.

Vandenbosch, L., Beyens, I., Vangeel, L., \& Eggermont, S. (2016). Online communication predicts Belgian adolescents' initiation of romantic and sexual activity. Eur J Pediatr. 175(4), 509-16.

Viana, T. B. P., et al. (2018). Factors associated with cigarette smoking among public school adolescents. Rev. esc. enferm. USP. 52:e03320.

White, C. N., \& Warner, L. A. (2015). Influence of Family and School-Level Factors on Age of Sexual Initiation. Journal of Adolescent Health, 56(2), 231237.

Young, H., Burke, L., \& Nic Gabhainn, S. (2018). Sexual intercourse, age of initiation and contraception among adolescents in Ireland: findings from the Health Behaviour in School-aged Children (HBSC) Ireland study. BMC Public Health. 18(1), 2-17.

Zito, R. C., \& Coster, S. (2016) Family Structure, Maternal Dating, and Sexual Debut: Extending the Conceptualization of Instability. Journal of Youth and Adolescence. 45(5), 1003-1019. 